\title{
Evaluation of a geometry-based knee joint compared to a planar knee joint
}

\author{
Anders Sandholm • Cédric Schwartz • Nicolas Pronost • \\ Mark de Zee • Michael Voigt · Daniel Thalmann
}

Published online: 29 January 2011

(C) Springer-Verlag 2011

\begin{abstract}
Today neuromuscular simulations are used in several fields, such as diagnostics and planing of surgery, to get a deeper understanding of the musculoskeletal system. During the last year, new models and datasets have been presented which can provide us with more in-depth simulations and results. The same kind of development has occurred in the field of studying the human knee joint using complex three dimensional finite element models and simulations. In the field of musculoskeletal simulations, no such knee joints can be used. Instead the most common knee joint description is an idealized knee joint with limited accuracy or a planar knee joint which only describes the knee motion in a plane. In this paper, a new knee joint based on both equations and geometry is introduced and compared to a common clinical planar knee joint. The two kinematical models are analyzed using a gait motion, and are evaluated using the muscle activation and joint reaction forces which are compared to invivo measured forces. We show that we are able to predict the lateral, anterior and longitudinal moments, and that we are able to predict better knee and hip joint reaction forces.
\end{abstract}

Keywords Knee joint - Inverse kinematics and dynamics . Joint reaction - Computed muscular control · OrthoLoad . Validation $\cdot$ Musculoskeletal model

A. Sandholm $(\bowtie) \cdot$ N. Pronost · D. Thalmann

Virtual Reality Lab., École Polytechnique Fédérale de Lausanne,

Lausanne, Switzerland

e-mail: anders.sandholm@epfl.ch

C. Schwartz $\cdot$ M. de Zee $\cdot$ M. Voigt

Center for Sensory-Motor Interaction, Aalborg University,

Aalborg, Denmark

N. Pronost

Games and Virtual Worlds, Utrecht University, Utrecht, The

Netherlands

\section{Introduction}

Today the field of neuromuscular simulations is widely used to understand the underlying dynamics of the movement of living beings, from gait research and treatment of patient with gait problems, to the teaching of physicians and the development of ergonomic furniture. During the last few years several platforms have been developed from commer-
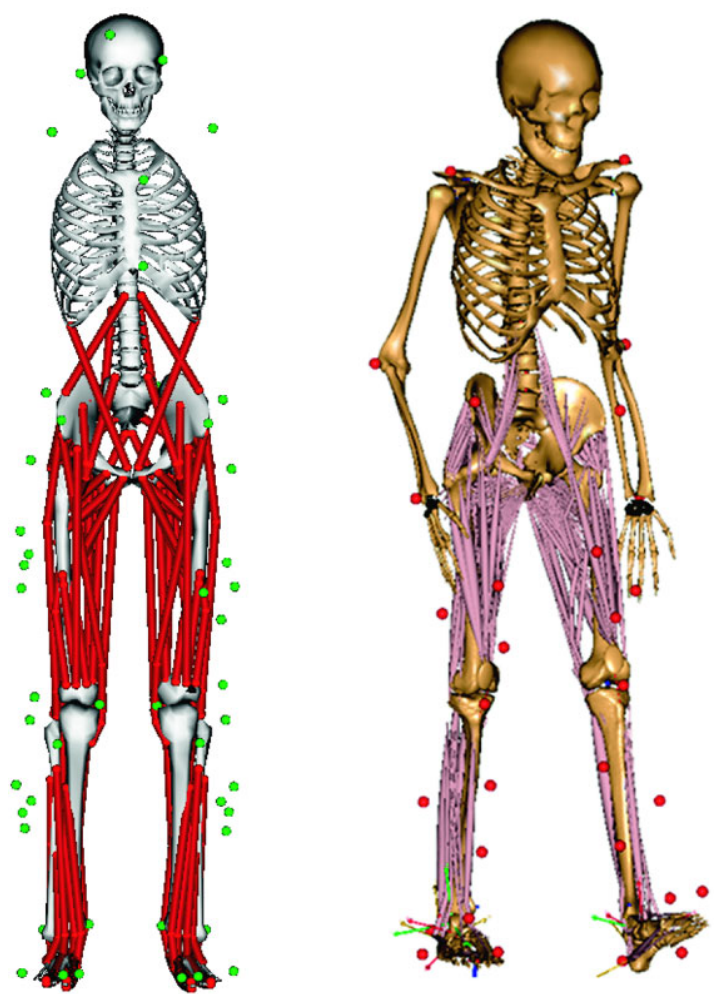

Fig. 1 (Left) OpenSim model with planar knee joint scaled into subject; (Right) AnyBody model with the geometry knee joint 
cial tools [6] to open-source based solutions [8]. The expansion of this field has also allowed the accessibility to musculoskeletal models that are able to describe different levels of complexity [3, 7, 32]. This development has given researchers and physicians powerful tools to analyze advanced simulations and even to execute 'what-if' scenarios before clinical treatment has even started. A problem with most of these models is that the human knee joint description is still rather undeveloped. This comes from the underlying complexity of the knee. Whereas the hip joint can be described during normal gait as a standard ball-socket joint with no translations and the ankle-subtalar joint as two revolute joints, the knee joint motion is much more complex including both translations and rotations during normal gait motions. In daily activity, such as normal non-pathological gait, the human knee joint is subjected to significant loads with peak values well-above the subject's body weight $[2,9$, $12,14,21]$. These load patterns are very complex because of several external forces exist (ground reaction force, mass and acceleration forces from the foot and the shank) which are counterbalanced by the forces acting inside the joint, such as the tibio-femoral contact forces and forces generated by the muscles to either keep the balance or to generate a motion. There are also smaller forces acting inside the joint such as soft tissue constraints, contact forces and forces created by internal friction. Due to this complexity, the knee joint has mostly been modeled and simulated using finite element method (FEM), which has been used in great success to analyze joint kinematics and a variety of problems relating to the knee joint $[10,11,24]$. In these models, the knee includes structures as ligaments and sophisticated materials $[23,31]$ which implement properties as transverse isotopy, nonlinear stress-strain curve and to a certain degree viscoelastic behavior. In musculoskeletal models, joints are not described as three dimensional meshes that are allowed to deform, instead joint kinematics are modeled as one or several functions controlled by one or several variables. This has led to the fact that in most common musculoskeletal models the human knee joint is either described as an idealized joint based only on rotations [15] or, as in most common clinical models, a planer knee joint [7]. In Delp et al. (1990), the knee joint is described using one degree of freedom (DoF) (knee flexion), with the additional two coupled DoFs, tibiofemoral translation and the nonsagittal rotation. A recent new knee model [3] was published, which uses one DoF (flexion) but with additional coupled dimensions to incorporate three translations and rotations of the tibia relative to the femur. This knee joint is based on tibiofemoral kinematics experimental measurements from 23 'normal-sized' adult knees $[20,30]$. In this paper, a new knee joint is presented which combines the geometric description from a finite element method and the functional description of musculoskeletal joints. The joint is based on the work of Walker et al. [30] and Kurosawa et al. [20]. The knee joint is defined as three DoFs, driven by flexion, with additional adductionabduction rotation and distration-compression translation described from the subject's knee geometry/anatomy.

\section{Material and methods}

In this study, a 34 year old male, weighing $89.6 \mathrm{~kg}$ with no recorded knee injury or other muscular or skeletal injuries was studied. Initially, an MRI acquisition (resolution: $0.39 \times 0.39 \times 1 \mathrm{~mm}$ ) of the subject's knee was performed. Both lateral and femoral knee condyles were segmented including cartilage (see Fig. 2(a)) [26, 27]. Gait motions were captured using eight cameras (Qualisys ProRe-

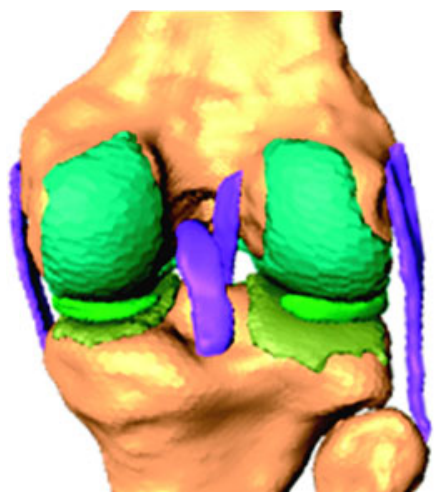

(a) Segmentation result of the knee joint in- (b) Condyles with each registered ellipsoid cluding cartilage and menisci.

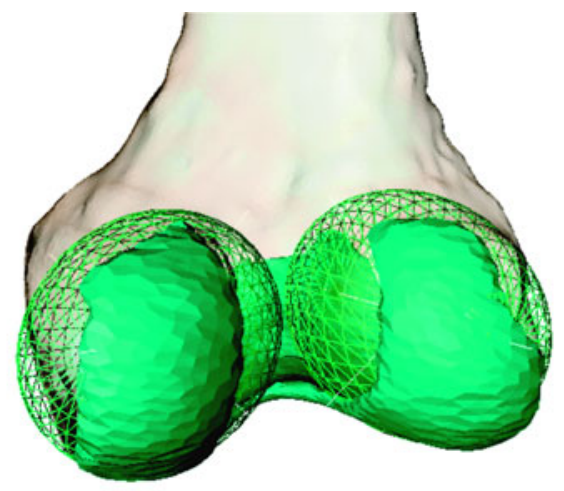

(c) Complete knee joint visualization. Contact points shown as violet spheres. The ellipsoids do not here aligned up with the femur because AnyBody visualize the femur with an generic model, not the subject specific femur used in the modelization.

Fig. 2 Stages of modeling the geometry knee joint 
flex, $200 \mathrm{~Hz})$ and two AMTI force platforms $(2000 \mathrm{~Hz})$. During the motion capture, eight electromyography sensors were used to record muscle activation in biceps femoris long head (BFLH), gastronemius (GAS), glutes maximus (GMAX), rectus femoris (RF), soleus (SOL), tibalius anterior (TA), vastus lateralis (VL), and vastus medialis (VM). During the motion capture, the Cleveland Clinic marker set was used [5], with additional four marker plates (each containing four reflective markers) placed on the lateral side of each thigh and shank. Kinematic and kinetic data along with EMG was extracted from C3D files using the MotionLab Matlab toolbox [25] and filtered using a third order Kalman filter [19] to ensure accurate an inverse kinematic solution [13]. The same data was exported to OpenSim and AnyBody formats. The subject kept a constant speed of $1.86 \mathrm{~m} / \mathrm{s}$ during the gait motion.

\subsection{Geometry-based knee joint}

To develop the geometry-based knee joint, the AnyBody Modeling System [6] was used. The geometry-based knee joint is defined to use knee flexion-extension from the subject's gait motion as the driving DoF. The other five DoF are defined depending on the position of the joint, adductionabduction rotation and distration-compression translation were constrained using the subject's knee anatomy, two (posterior-anterior translation and internal-external rotation) were driven by equations from Walker et al. [30] and Kurosawa et al. [20], the medial-lateral translation was locked.

The construction of these constraints was archived using three main steps:

1. Extraction of anatomical information

2. Information registration in the biomechanical model

3. Contact definition between the tibia and the femur

In the first step, a quadric robust-fitting approach [1] was used to model the condyles. The fitting methods resulted in two ellipsoids, one for each condyle (see Fig. 2(b)). The lateral and medial plateaus were described as two contact points which were determined as the closest points between the femoral and tibial prosthesis components. The second step involved the registration of the anatomical information in AnyBody: the knee model is based on the patient's anatomy represented as the ellipsoids and the contact points which were described in an AnyBody biomechanical model. The thigh segment (ellipsoids) registration was produced using the hip center and the two femoral epicondyles (see Fig. 3). The shank segment (contact points) registration was realized using the two ankle malleolars and the tibia tuberosity. In the third step, a model constraint was introduced to ensure contact between the lateral and medial ellipsoids and

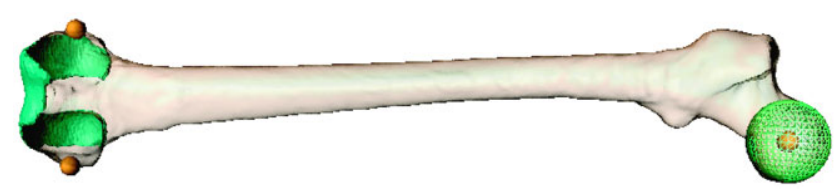

Fig. 3 Localization of the three landmarks used for the registration of the femur in the biomechanical model (yellow spheres)—medial and lateral epicondyles as well as the center of the femoral head, which was obtained after fitting a sphere (green mesh) on the articular surface of the femoral head

two points located respectively on the lateral tibial plateau and the medial tibial plateau (see Fig. 2(c)). To model the displacement that occurs in the knee during gait [16], the geometry-based knee joint also includes posterior-anterior translation and internal-external rotation given by the equations provided in Walker et al. [30]. Only the linear term was modeled and applied to the contact points relatively to the tibia segment.

\subsection{Planar knee joint}

The second knee model that we used in this study is the Yamaguchi et al. model [32] which is described as a planar knee joint which works in a plane using one DoF. The motion of the knee joint is represented by a pathway for the center of rotation that gives realistic orientations of the femur relative to the tibia. The joint uses two additional coupled DoF (posterior-anterior and distration-compression), described as functions of one rotational degree of freedom (knee flexion). These two relationships give the rolling-tosliding ratio of the motion of the femoral condyles on the tibial plateau.

\subsection{A common model}

In order to evaluate the geometry-based knee joint and to compare result to the planar knee joint a common neuromuscular model and simulation platform must be used. Therefore, a common model [7] was agreed upon consisting of a torso (including head) modeled as a ridged element with three rotational DoF relative to the pelvis. The pelvis could rotate and translate in all three dimensions. The hip joint was modeled as a ball-socket joint with three rotation DoF. The ankle-subtalar complex was represented by two revolute joints aligned with anatomical axes. The metarsophalangeal joint was modeled as a one DoF hinge joint which allowed flexion and extension. In both systems, the model was scaled to a subject-specific geometry that was based on the markers' position during a static trial which was followed by an inverse kinematic trial where joint angles and translations were determined. Both models were consistent regarding body elements and marker weights. Only the right knee joint was different. To be able to express the geometry-based 


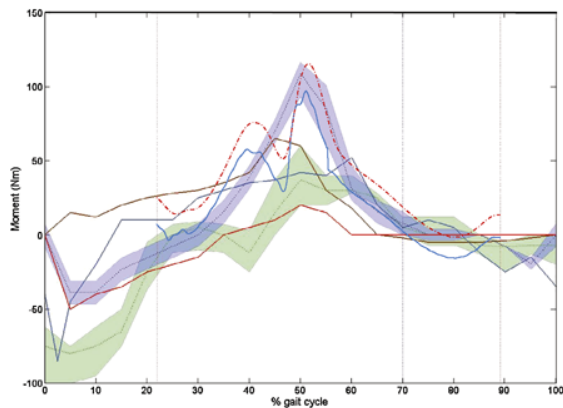

(a) Flexion

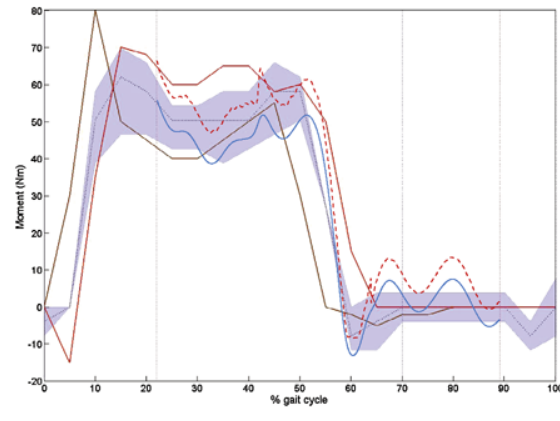

(b) Adduction

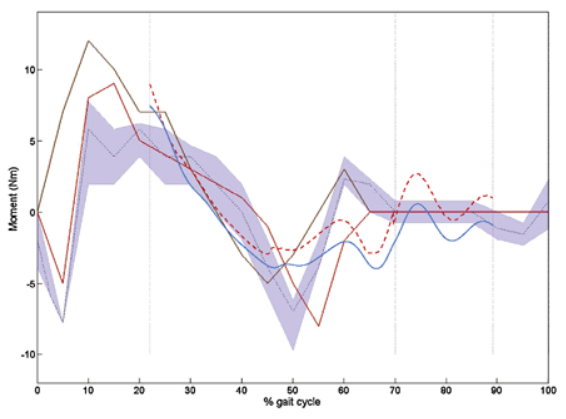

(c) Rotation
Fig. 4 Moment working on the hip joint from inverse dynamics. Blue solid line represents moment from geometry knee joint, while red dashed line shows moment from planar knee joint. Cappozzo et al. (1975) - green area, mean value (dotted green line); Patriarco et al.

knee joint kinematic in OpenSim, a new model was created using a free six DoF knee joint. To translate the geometrybased knee joint kinematic to the new OpenSim model, a common coordinate system was created for each body part using motion capture markers. Using these common coordinate systems for each frame of kinematic solution, a translation and rotation matrix was calculated which translated the femur and tibia kinematics from the Anybody system into OpenSim coordinate system. The neuromuscular models used to estimate muscle forces consisted of 92 muscle actuators [8]. In Fig. 1 the complete AnyBody model including the geometry based knee joint is shown, alongside the planar knee joint implemented in OpenSim.

\subsection{Dynamic simulation}

To investigate both knee joints and their ability to predict forces, OpenSim 2.0 was used. First, inverse dynamics were used to study the generalized forces acting upon each joint. Second, computed muscular control (CMC) [29] was used to find the optimal muscle excitation pattern that would drive the models along the desired trajectories. Third, a joint reaction analysis was carried out that calculated forces and moments acting on the hip and knee joint using the muscle forces estimated during CMC. During the simulations, both models used the same marker weight/cost functions and optimization values. The results from all three simulations were extracted using MotionLab and normalized over the gait cycle. EMG signals were extracted, normalized to the gait cycle, then high-pass filtered $(20 \mathrm{~Hz})$, rectified and finally low-pass filtered $(6 \mathrm{~Hz})$.

\section{Results}

To evaluate the geometry, knee joint results will be compared to the commonly used planar knee description. Results from inverse dynamics, computed muscular controls (1981)—brown line; Cowninshield et al. (1978)—red solid line; Kadaba et al. (1989) - blue area, mean value (dotted line); Inman et al. (1947)_dark blue line

and joint reactions from both the hip joint and knee joint will be presented using both knee joint models.

\subsection{Inverse dynamics}

Inverse Dynamic is a common way to investigate the generalized forces that act upon joints given a specific motion. This is done by using information from the model, such as body mass, inertia and kinematics (acceleration) to solve the equation of the second fundamental law of motion. In Fig. 4(a), hip joint flexion moment is shown. The literature data have been normalized to the subject's body weight and leg length and shown in Newton meters (Nm). Both knee joints show similar result with the data published by Kadaba et al. [18]. The planar knee joint model predicts a hip flexion moment that has a mean of $12 \mathrm{Nm}$ larger than the value predicted by the geometry-based knee joint model. Hip adduction moment (Fig. 4(b)) corresponds also with the data published by Kadaba et al. (1989). The planar knee joint model produces a mean value which is $8 \mathrm{Nm}$ larger than the geometry-based knee joint model. Figure 4(c) shows hip rotation moment, both the geometry-based knee joint model and the planar knee joint model show resemblance with the data published by Kadaba et al. (1989). The planar knee joint model estimates a moment on average larger by 2.2 Nm compared to the geometry-based knee joint model. Both knee joints display an oscillation starting at around $60 \%$ of the gait cycle and continue during the swing phase. This comes from the subject's foot motion during the toe-off and swig phase.

Inverse dynamics results for knee flexion are shown in Fig. 4(a). Here clear differences between the geometrybased knee joint and the planar knee joint model are present. The geometry-based knee joint displays the same trend as data published by Kadaba et al. (1989), while during the stance phase the planar knee joint corresponds to data published by Cappozzo et al. (1975), and during the swing phase 


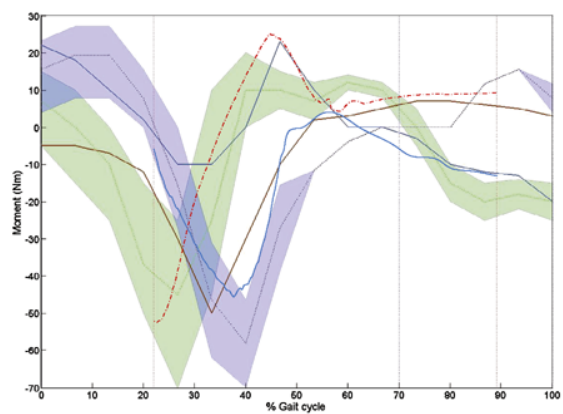

(a) Knee Flexion

Fig. 5 (a) Moment working on the hip joint from inverse dynamics. Blue solid line shows moment from geometry knee joint, red dashed line denotes moment from planar knee joint. Cappozzo et al. (1975)_ green area, mean value (dotted green line); Patriarco et al.

it correspond to data published by Patriarco et al. (1981). The mean difference between the planar knee joint and the geometry-based knee joint is $58 \mathrm{Nm}$. The largest contribution to the mean difference comes from the two trends shown during the stance phase. The planar knee joint model reaches its maximum value at $22 \%$ of the gait cycle while the geometry-based knee joint first reaches its maximum value at $40 \%$ of the gait cycle. Figure 5(b) shows the moment forces for adduction and rotation. These dimensions are not present in the planar knee joint, and therefore no moments are calculated for them. Also no literature data has been published for knee adduction/rotation moment forces.

\subsection{Muscle estimation}

Computed muscular control activation patterns from both knee models (Fig. 6) were in general consistent with the captured EMG pattern, although there are clear differences between the two knee joint models. In Fig. 6, the grey area represents \pm 1 standard deviation of the EMG signal calculated from 28 gait motions, which were all acquired during the same motion capture session.

For the hip extender/abductor GMAX (Fig. 6(c)), small differences in activation pattern exist, the planar knee joint model predicts a slightly higher activation value than the geometry-based knee joint. For VM (Fig. 6(h)) which is a knee extender, two patterns are present. First, the geometrybased knee joint model displays a better prediction of activation during the stance phase. Second, the planar knee joint model predicts an activation in VM starting at $80 \%$ of the gait cycle, this muscle activation is not present in the geometry-based knee joint model. The second knee joint extender VL (Fig. 6(g)) displays a large difference between the two knee joint models. The planar knee joint model predicts no activation for VL during the mid-stance phase, which the geometry-based knee joint model predicts. The

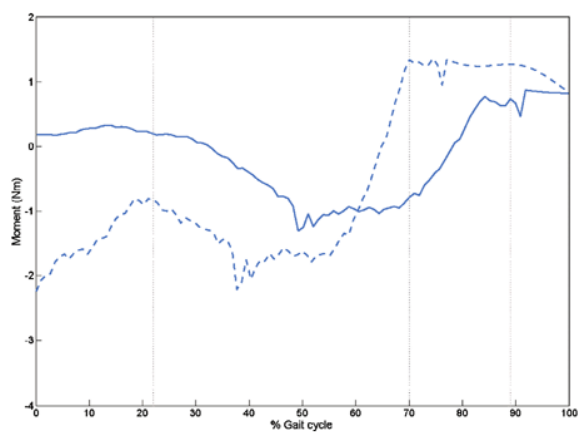

(b) Knee Adduction and Rotation

(1981)_brown line; Kadaba et al. (1989)—blue area, mean value (dotted line); Inman et al. (1947)—dark blue line. (b) Knee rotation (solid line), knee adduction (dashed line)

planar knee joint model also shows an activation before toeoff in VL, which is also slightly present in the geometrybased knee joint. For the muscles spanning over the ankle joint, some differences exist between the two models. SOL (Fig. 6(e)) shows a similar activation pattern for the two knee joint models. For TA (Fig. 6(f)), the models predict different activation during the stance-phase. The EMG pattern for TA shows a large activation during heel-strike which is deactivated around $10-15 \%$ of the gait cycle. In the planar knee joint model, this activation is still present until 34\% of the gait cycle. The geometry-based knee joint model shows no activation from $22 \%$ of the gait cycle were the CMC simulation starts in this study. For the muscle spanning both the hip joint and the knee joint, BFLH (Fig. 6(a)) and RF (Fig. 6(d)), similar differences are seen in the patterns between the two knee models. The geometry-based knee joint model predicted a lower activation during the toe-off than the planar knee joint model. The planar knee joint model predicts a higher activation of the RF after toe-off, which is not present in the captured EMG. For the muscle GAS (Fig. 6(b)) which spans over both the knee joint and the ankle joint, a clear difference between the models is present. The planar knee joint model predicts a mean of $24 \%$ higher activation until $50 \%$ in the gait cycle and then $10 \%$ higher activation until $60 \%$ in the gait cycle. From $60 \%$ and during toe-off, the planar knee joint model predicts a higher activation on average by $12 \%$ than the geometry-based knee joint model.

From the CMC result, it is clear that muscles spanning over the hip, knee and ankle joints are all affected by different knee joint descriptions.

\subsection{Joint reaction}

To better understand the difference between the two knee models and their effect on hip and knee joint forces, Open- 


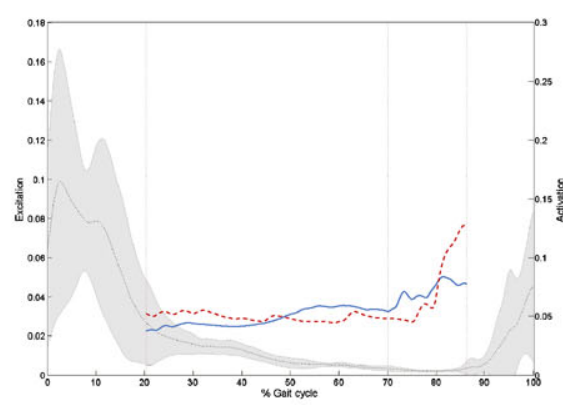

(a) Biceps femoris long head

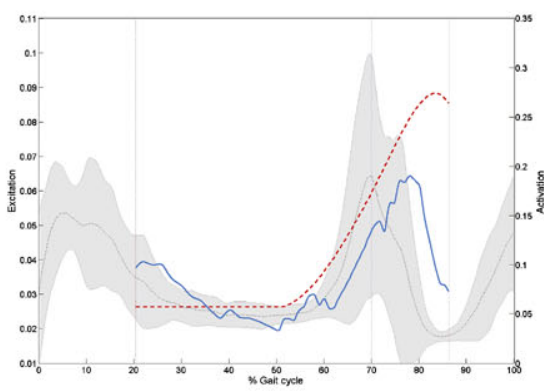

(d) Rectus femoris

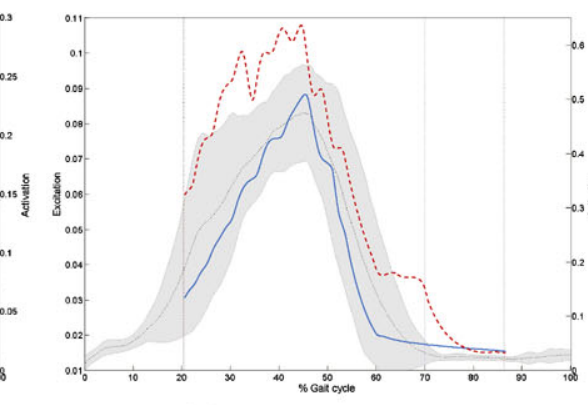

(b) Gastronemius

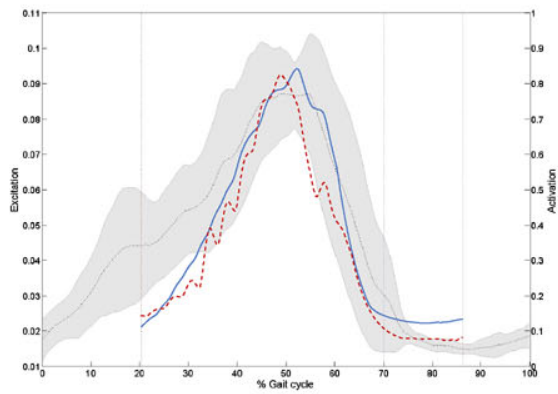

(e) Soleus

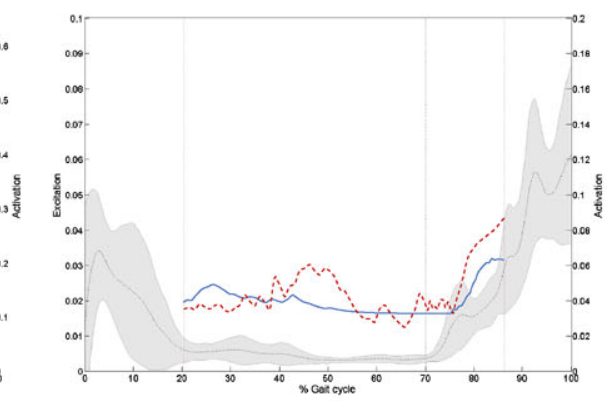

(c) Glutes maximus (lateral)

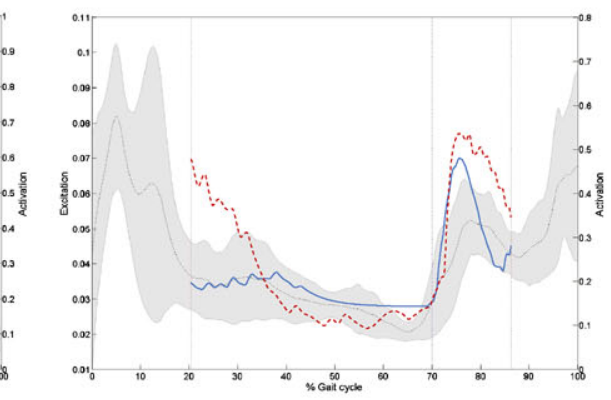

(f) Tibalius anterior

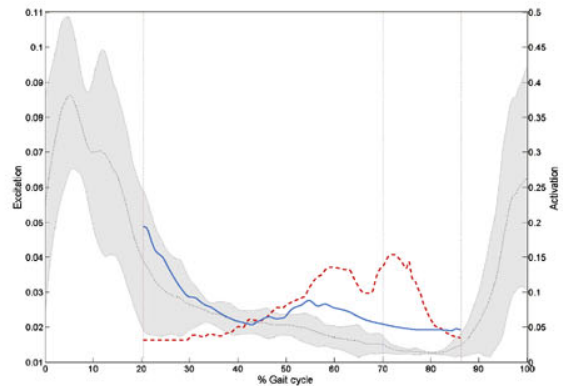

(g) Vastus lateralis

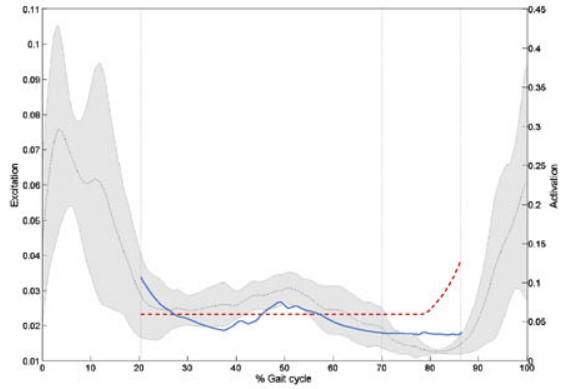

(h) Vastus medialis

Fig. 6 Comparison of experimental and simulated muscle activity between the geometry knee joint (blue solid line) and the planar knee joint (red dashed line). The shaded area represents \pm 1 standard deviation of the EMG using 28 gait motions

Sim was used to calculate the joint reaction using the muscle forces calculated during CMC (see Sect. 3.2). In order to evaluate the calculated joint forces, they were compared to data from OrthoLoad [4]. For the hip joint, five gait motions from four subjects (RHR, HSR, EBR and EBL) were extracted. The subjects were walking on a treadmill with a constant speed of $2 \mathrm{~m} / \mathrm{s}$. For the knee joint, seven gait motions were downloaded from four subjects (K1L, K3R, K4R and K5R). The subjects walked on a planar floor at the own speed. To process the data, a full gait cycle was identified using the synchronized video sequence. The force data were then imported into MotionLab, normalized over the gait cycle, and a standard deviation of \pm 1 was calculated for each joint and dimension. Joint reaction forces for the hip joint are shown in Fig. 7 alongside \pm 1 standard deviation of the in-vivo OrthoLoad forces. Forces calculated from joint reaction were normalized to $\% \mathrm{BW}$ and moments to $\% \mathrm{BW} * \mathrm{~m}$.
In the superior hip joints direction (Fig. 7(a)), both knee joint models show clear difference in force load. In the midstance phase, from $30 \%$ to $40 \%$ of the gait cycle, the planar knee joint model predicts a decrease in superior force with a mean value of $62 \% \mathrm{BW}$ compared to the geometry-based knee joint model. The second differences are between 55\% to $70 \%$ of the gait cycle, were the planar knee joint model shows an increase in superior force with a mean value of $60 \% \mathrm{BW}$ compared to the geometry-based knee joint model. Except for these two phases, both models predict similar forces in the superior hip joint direction as presented in the OrthoLoad data. In the lateral direction of the hip joint (Fig. 7(b)), both the geometry-based knee joint model and the planar knee joint model display oscillations in the force estimation. This oscillation is more prominent in the planar knee joint model which also produced a higher force value with a mean difference of $19 \% \mathrm{BW}$. In the lateral direction 


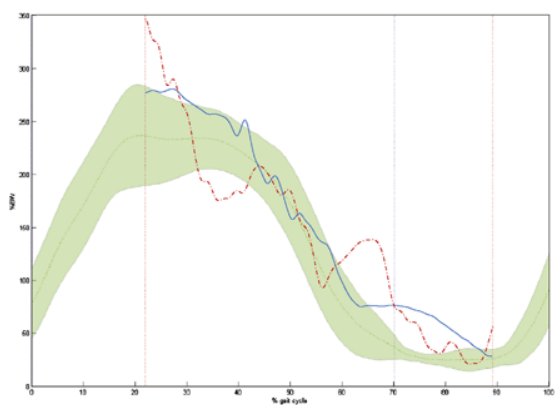

(a) Superior hip force

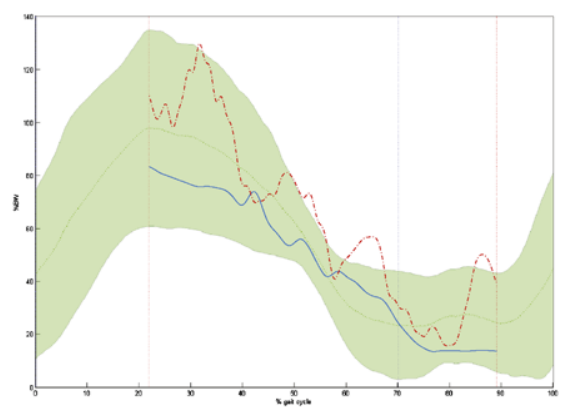

(b) Lateral hip force

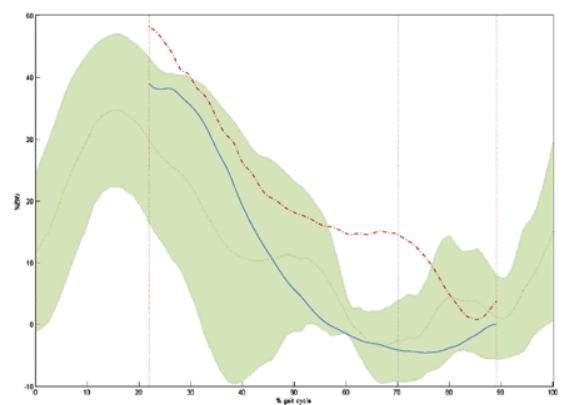

(c) Anterior hip force
Fig. 7 Comparison of in-vivo measured hip joint forces and simulated joint reaction forces, geometry-based knee joint visualized as blue solid line, planar knee joint visualized as red dashed line. Green shaded area represents \pm 1 standard deviation of in-vivo measured hip forces from the OrthoLoad database

moment (Fig. 8(d)) and superior moment (Fig. 8(f)) display the same pattern. In both directions, the planar knee predicts a higher moment during both the stance and the swing phase. For the superior moment, the planar knee joint shows a higher mean value of $1.1 \% \mathrm{BW}^{*} \mathrm{~m}$ and in the lateral direction a higher mean value of $1.3 \% \mathrm{BW}^{*} \mathrm{~m}$. In the lateral direction, the geometry-based knee joint shows the same pattern as the OrthoLoad data, except during the swing phase were a higher value is predicted. In the superior direction, neither of the knee joints shows a good match to the OrthoLoad data. During the first part of the gait cycle, the planar knee joint shows a better prediction than the geometry-based knee joint. During the later part of the gait-cycle, the geometrybased knee joint predicts a closer match to the OrthoLoad data. In the knee's anterior direction (Fig. 8(b)), both the planar knee joint and the geometry-based knee joint predicted a smaller value than OrthoLoad. The anterior moment given by the geometry-based knee joint model resembles the pattern from OrthoLoad data, the planar knee model shows almost a constant value.

In general, we can see that the planar knee joint produces higher loads in both the hip joint and the knee joint than the geometry-based knee joint model. The geometry-based knee joint model also predicts forces closer to the forces present in the OrthoLoad data.

\section{Discussion}

The use of neuromuscular models and simulations has given clinicians and researchers a tool to look deep into the functions of muscles, joints and bones and their effects on movability. Due to the complexity of these dynamic systems, we must have absolute confidence in the accuracy of models and simulations before any decisions or conclusions are made. Most often when a new model is presented the results are validated against EMG. The use of surface EMG can provide some insight into muscles behavior, but it can only valWhen it comes to moment forces in the knee joint, the lateral 
Fig. 8 Comparison of in-vivo measured knee forces and simulated joint reaction forces, geometry-based knee joint visualized as blue solid line, planar knee joint visualized as red dashed line. The shaded area represents \pm 1 standard deviation of in-vivo measured knee forces from the OrthoLoad database

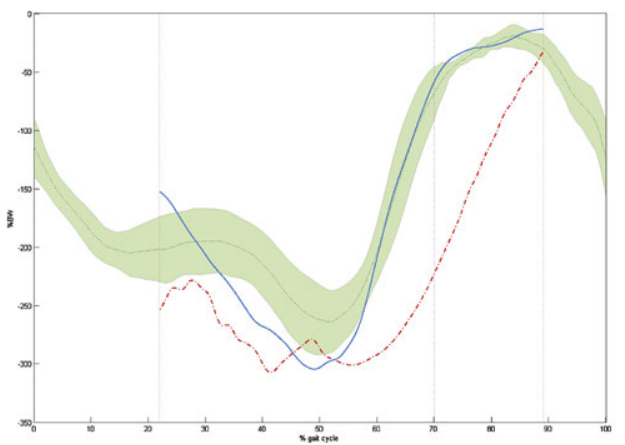

(a) Anterior knee forces

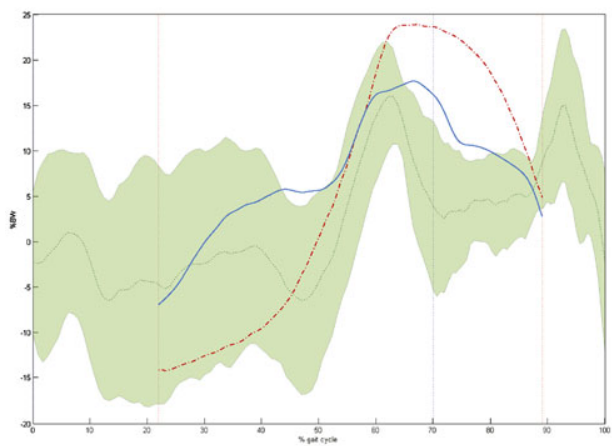

(c) Lateral knee force

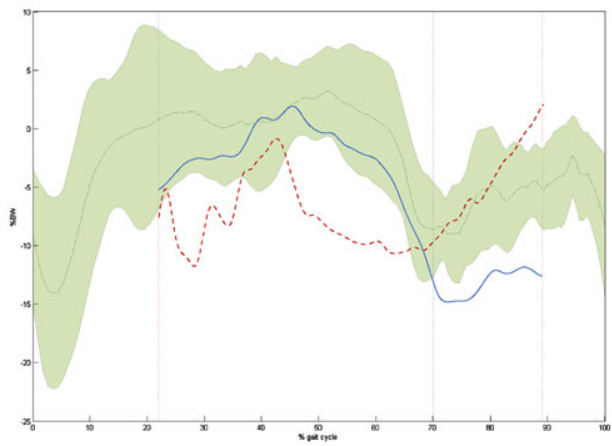

(e) Superior knee force

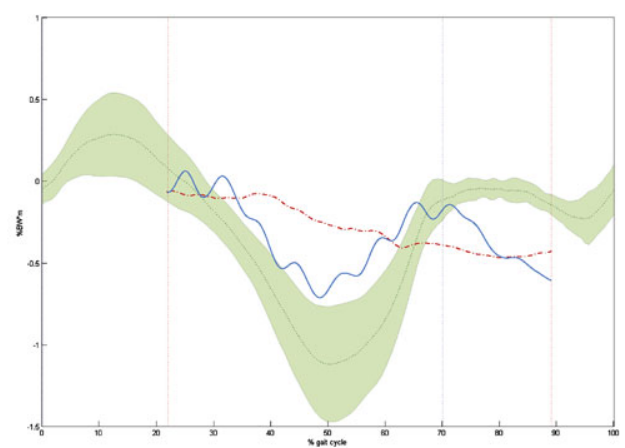

(b) Anterior knee moment

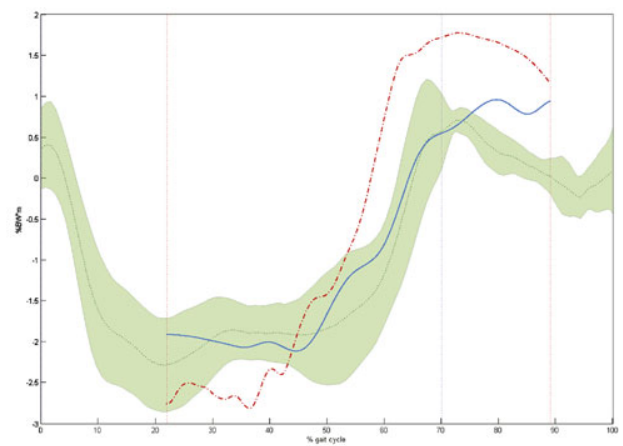

(d) Lateral knee moment

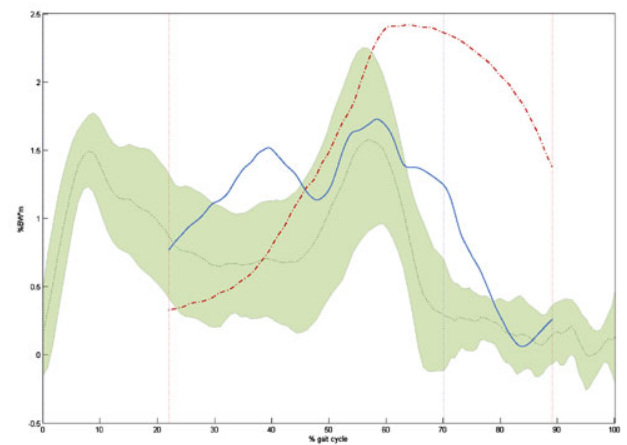

(f) Superior moment idate a small set of muscles located close to the surface of the skin.

We believe that this is not enough when it comes to validating new models or evaluating new joints' dynamics. Therefore, we believe that it is absolutely necessary to evaluate using joint reaction forces which values incorporate all muscles spanning over a joint. These joint reaction forces can then be compared to forces measured in-vivo, like data from the OrthoLoad project. The presented study constitutes such a validation methodology, and provides further insight into the complexity of the human knee joint. To ensure an accurate kinematic solution, great care were taken to minimize skin movements and other artifacts which can heavily effect inverse kinematic solutions. The two models used were identical expect that in one model the geometry-based knee joint (see Sect. 2.1) was used and in the other the planar knee joint (see Sect. 4).
The first validation used in this study was inverse dynamics of the hip joint and the knee joint which were compared to the literature data (Figs. 4 and 5). The use of inverse dynamics provides a powerful tool to evaluate a model, but it does have some limitations. First, inverse dynamics assumes that there is no friction inside the joint and that the mass distribution is uniform in each segment. Another source of error is the misplacement of joint centers which alters the direction a body segment is accelerated. When solving the inverse dynamics problem, an iterative process is used, which starts from the ankle joint and propagates up in the model. Therefore, errors that are introduced in the ankle joint solution propagate to the knee joint which errors propagate to the hip joint, and so forth. In the published inverse dynamics literature, data large differences exist between the studies. For the knee joint Kadaba et al. (1989) and Cappozzo et al. (1975) predicts two different locations for the max- 
imum knee flexion moment. The same literature data also predict two different patterns for the swing phase. For the hip joint, uniform pattern is shown for adduction and rotation except for a small differences in value. For hip flexion, large differences exist between the different studies, both in value and pattern. The inverse dynamics result presented using both models shows clearly that the knee joint description affects both the knee moment and the hip moment. All segments in both models were equal in lengths/weights and inertia matrixes. Therefore, the different inverse dynamics solutions can only come from the different kinematics each knee joints produced and differences in the joint center.

To better evaluate the differences shown in the inverse dynamics results, computed muscular controls were used to investigate both knee joints' models and their effect on the model to produce the necessary muscle force needed to track the desired kinematics. The calculated muscle activation (Fig. 6) corresponds to activation levels and patterns published in other gait studies [22]. For both knee joints, the predicted activation agreed with the captured EMG; however, for some muscles, a clear difference existed. A common trend could be seen in the estimated muscle activations. For the geometry-based knee joint model, lower muscle activation is needed to track the kinematic solution than if the planar knee joint model is used. A larger difference can be seen in the GAS, RF and TA muscles that span all three joints in the lower limb. GAS and TA show large differences in activation during the mid-stance phase, while the RF shows a large difference during the swing phase. For VM, BFLH, VL and SOL only minor differences exist between the two knee models. To deeper investigate the difference between the knee models, joint reaction forces were estimated (Figs. 7 and 8) and compared to forces available from the OrthoLoad database. However, the numbers of subject and motions available are too few to do any statistical analysis between the in-vivo forces and predicted model forces. Instead, the \pm 1 standard deviation calculated from OrthoLoad can only be used as an estimate of the invivo knee and hip joint forces. Both knee models predicted higher forces and moment than the OrthoLoad data showed. This overestimation of forces has been shown in other studies $[17,28]$ where results from neuromuscular models have been compared to in-vivo measurements. However, some clear trends can be seen from this study. For the hip joint, the geometry-based knee joint predicts values that lie inside the \pm 1 standard deviation, while the planar knee joint predicts much higher forces. This implies that forces predicted in the hip joint are highly dependent on the knee joint description. The same trend is present for both knee joints, the planar knee joint predicts larger knee forces in anterior, lateral and superior direction than the geometry-based knee joint or OrthoLoad in-vivo forces.

In this paper, a new geometry-based knee joint model is presented and compared to in-vivo measurements. The result presented clearly shows that a geometry-based knee joint predicts better muscle activation and joint reaction forces than a planar knee joint. However, this should be seen in the light of two limitations. Only one subject has been investigated so far and for this subject only one gait motion is included in this study. Investigations using more subjects and motions are currently performed.

\section{Conflict of interest statement}

There are no conflicts of interest.

Acknowledgements This work is supported by the European Marie Curie Program under the 3D ANATOMICAL HUMAN project (MRTN-CT-2006-035763). Motion capture was carried out at SMI, Aalborg University. MRI acquisitions were carried out at UCL under supervision of Prof. Andrew Todd-Pokropek and Mitchelle Cheng.

\section{References}

1. Allaire, S., Jacq, J.-J., Burdin, V., Roux, C.: Ellipsoid-constrained robust fitting of quadrics with application to the $3 \mathrm{~d}$ morphological characterization of articular surfaces. In: Engineering in Medicine and Biology Society, 2007. EMBS 2007. 29th Annual International Conference of the IEEE, Aug. 2007, pp. 5087-5090 (2007)

2. Anderson, F.C., Pandy, M.G.: Static and dynamic optimization solutions for gait are practically equivalent. J. Biomech. 34(2), 153$161(2001)$

3. Arnold, E., Ward, S., Lieber, R., Delp, S.: A model of the lower limb for analysis of human movement. Ann. Biomed. Eng. 38, 269-279 (2010). doi:10.1007/s10439-009-9852-5

4. Bergmann, G., Deuretzbacher, G., Heller, M., Graichen, F., Rohlmann, A., Strauss, J., Duda, G.N.: Hip contact forces and gait patterns from routine activities. J. Biomech. 34(7), 859-871 (2001)

5. Castagno, P., Richards, J., Freenan, M., Lennon, N.: Comparison of 3-dimensional lower extremity kinematics during walking gait using two different marker sets. Gait Posture 3(2), 87-87 (1995)

6. Damsgaard, M., Rasmussen, J., Christensen, S.T., Surma, E., de Zee, M.: Analysis of musculoskeletal systems in the anybody modeling system. Simul. Model. Pract. Theory 14(8), 1100-1111 (2006). SIMS 2004

7. Delp, S.L., Loan, J.P., Hoy, M.G., Zajac, F.E., Topp, E.L., Rosen, J.M.: An interactive graphics-based model of the lower extremity to study orthopaedic surgical procedures. IEEE Trans. Biomed. Eng. 37(8), 757-767 (1990)

8. Delp, S.L., Anderson, F.C., Arnold, A.S., Loan, P., Habib, A., John, C.T., Guendelman, E., Thelen, D.G.: Opensim: Open-source software to create and analyze dynamic simulations of movement. IEEE Trans. Biomed. Eng. 54(11), 1940-1950 (2007)

9. DLima, D.D., Patil, S., Steklov, N., Chien, Shu, Colwell, C.W. Jr.: In vivo knee moments and shear after total knee arthroplasty. J. Biomech. 40(Suppl. 1), S11-S17 (2007). Interaction of Mechanics and Biology in Knee Joint Restoration and Regeneration

10. Ellis, B.J., Lujan, T.J., Dalton, M.S., Weiss, J.A.: Medial collateral ligament insertion site and contact forces in the acl-deficient knee. J. Orthop. Res. 24(4), 800-810 (2006)

11. Fernandez, J., Hunter, P.: An anatomically based patient-specific finite element model of patella articulation: towards a diagnostic tool. Biomech. Model. Mechanobiol. 4, 20-38 (2005). doi:10.1007/s10237-005-0072-0 
12. Glitsch, U., Baumann, W.: The three-dimensional determination of internal loads in the lower extremity. J. Biomech. 30(11-12), 1123-1131 (1997)

13. De Groote, F., De Laet, T., Jonkers, I., De Schutter, J.: Kalman smoothing improves the estimation of joint kinematics and kinetics in marker-based human gait analysis. J. Biomech. 41(16), 3390-3398 (2008)

14. Heinlein, B., Kutzner, I., Graichen, F., Bender, A., Rohlmann, A., Halder, A.M., Beier, A., Bergmann, G.: Complete data of total knee replacement loading for level walking and stair climbing measured in vivo with a follow-up of 6-10 months. Clin. Biomech. 24(4), 315-326 (2009)

15. Horsman, K.: The Twente lower extremity model. $\mathrm{PhD}$ thesis, Department of Engineering Technology, University of Twente, Netherlands (2007)

16. Moro-oka, T.A., Hamai, S., Miura, H., Shimoto, T., Higaki, H., Fregly, B.J., Iwamoto, Y., Banks, S.A.: Dynamic activity dependence of in vivo normal knee kinematics. J. Orthop. Res. 26(4), 428-462 (2007)

17. Morrison, J.B.: Function of the knee joint in various activities. Biomed. Eng. 4, 573-580 (1969)

18. Kadaba, M.P., Ramakrishnan, H.K., Wootten, M.E., Gainey, J., Gorton, G., Cochran, G.V.: Repeatability of kinematic, kinetic, and electromyographic data in normal adult gait. J. Orthop. Res. 7(6), 849-860 (1989)

19. Kalman, R.E.: A new approach to linear filtering and prediction problems. Trans. ASME, J. Basic Eng. 82, 35-45 (1960)

20. Kurosawa, H., Walker, P.S., Abe, S., Garg, A., Hunter, T.: Geometry and motion of the knee for implant and orthotic design. J. Biomech. 18(7), 487-491, 493-499 (1985)

21. Kutzner, I., Heinlein, B., Graichen, F., Bender, A., Rohlmann, A., Halder, A., Beier, A., Bergmann, G.: Loading of the knee joint during activities of daily living measured in vivo in five subjects. J. Biomech. 43(11), 2164-2173 (2010)

22. Liu, M.Q., Anderson, F.C., Schwartz, M.H., Delp, S.L.: Muscle contributions to support and progression over a range of walking speeds. J. Biomech. 41(15), 3243-3252 (2008)

23. Pioletti, D.P., Rakotomanana, L.R., Benvenuti, J.F., Leyvraz, P.F.: Viscoelastic constitutive law in large deformations: application to human knee ligaments and tendons. J. Biomech. 31(8), 753-757 (1998)

24. Ramaniraka, N.A., Saunier, P., Siegrist, O., Pioletti, D.P.: Biomechanical evaluation of intra-articular and extra-articular procedures in anterior cruciate ligament reconstruction: a finite element analysis. Clin. Biomech. 22(3), 336-343 (2007)

25. Sandholm, A., Pronost, N., Thalmann, D.: Motionlab: a matlab toolbox for extracting and processing experimental motion capture data for neuromuscular simulations. In: Magnenat-Thalmann, N. (ed.) Modelling the Physiological Human. Lecture Notes in Computer Science, vol. 5903, pp. 110-124. Springer, Berlin (2009)

26. Schmid, J., Magnenat-Thalmann, N.: MRI bone segmentation using deformable models and shape priors. Med. Image Comput. Comput. Assist. Interv., 119-126 (2008)

27. Schmid, J., Sandholm, A., Chung, F., Thalmann, D., Delingette, H., Magnenat-Thalmann, N.: Musculoskeletal simulation model generation from MRI data sets and motion capture data. In: Magnenat-Thalmann, Nadia, Zhang, J.J.J., Feng, D.D.D. (eds.) Recent Advances in the 3D Physiological Human, pp. 3-19. Springer, London (2009)
28. Taylor, W.R., Heller, M.O., Bergmann, G., Duda, G.N.: Tibiofemoral loading during human gait and stair climbing. J. Orthop. Res. 22(3), 625-632 (2004)

29. Thelen, D.G., Anderson, F.C.: Using computed muscle control to generate forward dynamic simulations of human walking from experimental data. J. Biomech. 39(6), 1107-1115 (2006)

30. Walker, P.S., Rovick, J.S., Robertson, D.D.: The effects of knee brace hinge design and placement on joint mechanics. J. Biomech. 21(11), 965-967, 969-974 (1988)

31. Weiss, J.A., Maker, B.N., Govindjee, S.: Finite element implementation of incompressible, transversely isotropic hyperelasticity. Comput. Methods Appl. Mech. Eng. 135(1-2), 107-128 (1996)

32. Yamaguchi, G.T., Zajac, F.E.: A planar model of the knee joint to characterize the knee extensor mechanism. J. Biomech. 22(1), $1-10(1989)$

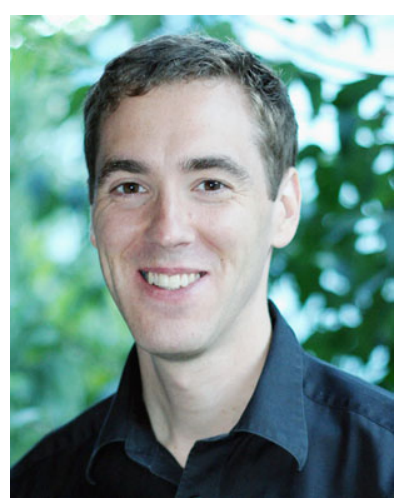

Anders Sandholm received his Ph.D. in 2011 from The Virtual Reality Lab, École Polytechnique Fédérale de Lausanne, Switzerland. His research topics included neuromuscular modeling, knee modeling and scientific visualization. He received his M.Sc. in Computer Science from University of Linköping, Sweden, were he focused on scientific visualization of large dataset. Prior to his Ph.D. research, he carried out a clinical study on a medical haptic catheter simulator at the E-health institute at Kalmar University. During this time, Anders also carried out a clinical study at Stanford University on how a Gait e-book can be used to assist clinicians in orthopedic diagnostics. Anders has also a background in information technology, both as a consultant in the financial industry and as a co-founder of two consulting companies. His main topics include knee joint description, gait simulation, neuromuscular models and using scientific visualization and information technology in order to extract knowledge from large datasets.

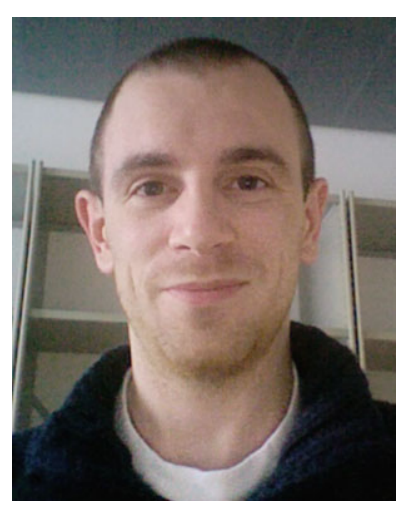

Cédric Schwartz received his $\mathrm{Ph} . \mathrm{D}$ degree in Physics and Engineering Science from TELECOM Bretagne and the Université de Bretagne Occidentale, within the Laboratory of Medical Information Processing (LaTIM, France) in 2009. After being a postdoc at Aalborg University (SMI Center, Denmark) involved in the biomechanical modeling of the knee, he is currently with the University of Liège (Laboratory of Human Motion Analysis, Belgium). His current research interests include the study of the asymmetrical motions of specialized athletes and their relation with pathological context. 


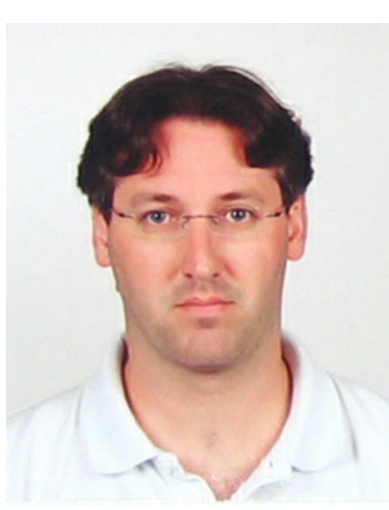

Nicolas Pronost is an Assistant Professor at Utrecht University, the Netherlands, in the Games and Virtual Worlds research group. He studied Computer Science at the University of Rennes 1, France, where he obtained his Ph.D. in 2006 and worked within the SIAMES/ Bunraku project of the INRIA/IRISA Laboratory. Then he was successively a postdoctoral researcher at the State Key Lab of CAD \& CG at Zheijiang University, China, and a postdoctoral researcher (Marie Curie Experienced Researcher) in the VRLab at EPFL (Switzerland). The main topics of his work are the analysis and the synthesis of human motions for biomechanical simulation and animation.

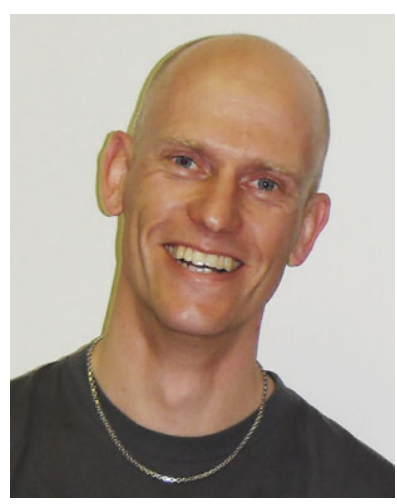

Mark de Zee is an Associate Professor at the Department of Health Science and Technology at Aalborg University in Denmark. He received his Master's degree in Mechanical Engineering in 1996 from the University of Twente in the Netherlands. In 2002, he received his Ph.D. degree in Biomedical Engineering from Aalborg University. He has been a part of the AnyBody Research Group since 2001, and he has more than 10 years of experience in developing and validating musculoskeletal models of different parts of the human body. He is one of the founders of the company AnyBody Technology A/S, and he is currently the vice-president of the Danish Society of Biomechanics.

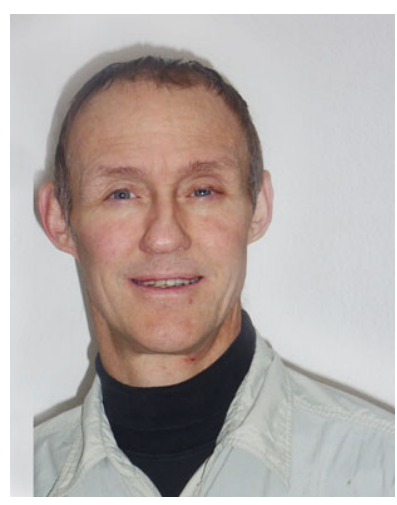

Michael Voigt received his M.Sc. degree in Human Physiology in 1987 and his Ph.D. degree in Biomechanics and Motor Control in 1994 from University of Copenhagen, Denmark. Since 1994, he worked at the Center for SensoryMotor Interaction at Aalborg University, Denmark, covering analysis of human motion and different aspects of muscle-tendon mechanics and its relation to motor control. He established the research group 'Physical Activity and Human Performance' and currently holds a professorship in Exercise Science at The Department of Health Science and Technology at Aalborg University. Dr. Voigt is a member of The International Society of Biomechanics.

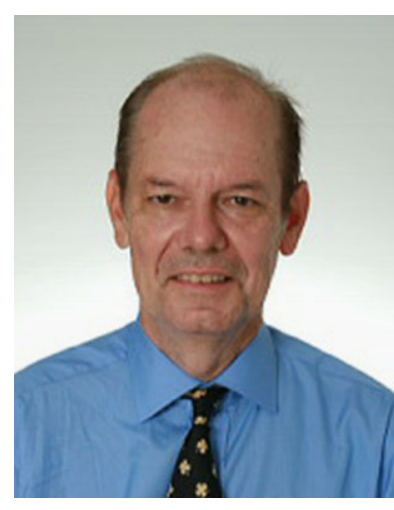

Daniel Thalmann is with the Institute for Media Innovation at the Nanyang Technological University in Singapore. He is a pioneer in research on Virtual Humans. His current research interests include Realtime Virtual Humans in Virtual Reality, crowd simulation, and 3D Interaction. Daniel Thalmann has been the founder of The Virtual Reality Lab (VRlab) at EPFL. He is a coeditor-in-chief of the Journal of Computer Animation and Virtual Worlds, and a member of the editorial board of 6 other journals. Daniel Thalmann was a member of numerous Program Committees, Program Chair and Cochair of several conferences, including IEEE VR, ACM VRST, and ACM VRCAI. Daniel Thalmann has published more than 500 papers in Graphics, Animation, and Virtual Reality. He is a coeditor of 30 books, and a coauthor of several books including "Crowd Simulation" and "Stepping Into Virtual Reality", published in 2007 by Springer. He received his Ph.D. in Computer Science in 1977 from the University of Geneva and an Honorary Doctorate (Honoris Causa) from University Paul Sabatier in Toulouse, France, in 2003. He also received the Eurographics Distinguished Career Award in 2010. 